\title{
Use of Ketamine in Ameliorating Opioid Withdrawal Symptoms During an Induction Phase of Buprenorphine
}

\author{
Sota Omoigui*, Fahad Hashmat and Zeudi Bernardo
}

\author{
L.A. Pain Clinic, 4019 W. Rosecrans Ave, Hawthorne, CA 90250, USA
}

\begin{abstract}
Ketamine can be used in the treatment of opioid withdrawal symptoms. Here are two case discussions of treatment of withdrawal symptoms in opioid dependent patients undergoing detoxification. Our case reports endorse the hypothesis that N-methyl D-aspartate (NMDA) antagonists may selectively inhibit the expression of opiate withdrawal. The use of intravenous ketamine can be considered as a bridge to successful initiation of buprenorphine to wean the patient off from high opioid doses while providing adequate analgesia during the transition period.
\end{abstract}

Keywords: Ketamine, opioid, opiate, withdrawal symptoms, buprenorphine.

\section{INTRODUCTION}

Opiate withdrawal occurs when a person dependent on opiates abruptly stops taking the drug. The symptoms of withdrawal last for approximately three days, and are not associated with life-threatening physiological changes. Side effects are very unpleasant, however, consisting of headaches, sweating, chills, muscle spasms, vomiting, and diarrhea. The exact sites in the brain that are involved in withdrawal from opiates have not yet been revealed. Some studies have suggested that the nucleus accumbens and the amygdala are regions activated during opiate withdrawal [1].

Ketamine is classified as a dissociative anesthetic and an NMDA receptor antagonist. It is frequently used for analgesia, and procedural sedation in pediatric patients and for the induction of general anesthesia in trauma patients. At the L.A. Pain Clinic, we utilize mini intravenous doses of Ketamine for procedural sedation, treatment of severe nociceptive and neuropathic pain as well as amelioration of opioid withdrawal and acute depressive symptoms. A common side effect is hallucinations. There have been few reports in the literature that administration of ketamine during withdrawal from opiates suppresses the expression of withdrawal symptoms [2].

Buprenorphine is a partial opiate agonist that helps patients to be weaned off from opioids by attaching to receptors bound by these opioids. Withdrawal symptoms are initially pronounced during the induction phase but start to get better as buprenorphine fills up these receptors. In addition, buprenorphine reduces cravings. Buprenorphine tightly attaches to the receptors and blocks other opioids from attaching. With adequate maintenance doses, buprenorphine fills most receptors completing a successful transition.

*Address correspondence to this author at the L.A. Pain Clinic, 4019 W. Rosecrans Avenue Hawthorne, CA 90250, USA; Tel: 310-675-9121;

Fax: 310-675-7989; E-mail: medicinechief@aol.com
We utilized the effects of subanesthetic doses of IV ketamine in adults as a bridge to treat opiate withdrawal in the induction phase of buprenorphine. When used in the acute phase of opiate withdrawal, ketamine can ease the symptoms and distress patients experience while being transitioned to buprenorphine.

\section{CASE REPORT \#1 - EC}

A 42 year old female with a history of polysubstance abuse, bipolar disorder, anorexia nervosa, and schizophrenia presented to the clinic for the first time in a state of sedation with complaints of severe low back pain, neck pain, headache, rhinorrhea, visual problems, and constipation. Further history revealed that her back pain was severe, 9 out of 10 on the pain scale, sharp in nature, radiating bilaterally to her lower extremities, and aggravated by activity.

The patient had a history of polysubstance abuse, using cocaine from the age of 11 to the age of 20 , when she was admitted into a centre for detoxification. She stated that she remained clean for 10 years, and then she began using cocaine again at the age of 30 , until she was 40 years old. She had a history of smoking marijuana for 25 years, and drinking alcohol excessively for 30 years. She stated that she had already taken 13 tablets of hydromorphone on the day of presentation prior to arriving at the clinic, and that she was enrolled in Alcoholics Anonymous. Her medications at that time included several drugs for pain, including hydromorphone, methadone, topiramate, and celecoxib, as well as psychiatric drugs such as aripiprazole, trazodone, and bupropion.

Upon physical examination, the patient was mentally confused, and disoriented to time, place, and person due to over-medication with opioids. Lumbar examination revealed severe tenderness over the levels of L4-S1, with decreased range of motion. An MRI of her cervical spine revealed multilevel disc desiccation with 1-2 mm annular bulge, mild impingement of the nerve roots, and stenosis. Lumbar MRI revealed disc desiccation from L1-L3, and L4-L5 as well, 
with a $3-4 \mathrm{~mm}$ disc protrusion with biforaminal stenosis at that level. The patient was recommended for discontinuation of methadone (10mg 3 times a day) and hydromorphone (4mg 4 times a day) and follow up in the clinic in 2 days, when she would be in a state of moderate withdrawal.

When the patient next presented to the clinic two days later, she was in withdrawal, experiencing intense symptoms of diarrhea, sweating, abdominal cramps, dizziness, lack of sleep, nausea, and vomiting. A Normal Saline infusion was initiated and the patient was given Ketamine $5 \mathrm{mg}$ IV bolus (repeated once), magnesium sulfate $3.5 \mathrm{~g}$ IV infusion, and ketorolac $60 \mathrm{mg}$ IV bolus to treat her symptoms. She was also started on the Suboxone (buprenorphine/naloxone) induction dose of 2-0.5 mg film - 2 films two times a day for the first day, then 2 films four times a day for the second day and thereafter. Six days later, in the follow-up visit, the patient reported having been free of withdrawal symptoms for 18 hours post IV treatment.

Before the IV ketamine, the withdrawal symptoms were severe and continuous, and afterward, the patient reported the symptoms being intermittent and far milder. The patient reported that the nausea and vomiting had stopped completely, and the frequency of diarrhea had decreased from 9 to 10 episodes per day to 3 to 4 episodes per day. Her pain also decreased from a level of 9 out of 10 on the pain scale to 5 out of 10 during the withdrawal period, which enabled her to manage herself more comfortably during her titration phase of Suboxone as she continued with treatment. She reported that the ketamine treatment had helped in relieving her withdrawal symptoms and her pain, and Suboxone helped decrease her body's physiological cravings from the discontinuation of her narcotic treatment. A follow up after three months since her first presentation showed that patient was doing well and not experiencing any withdrawal symptoms thus completing a successful transition to buprenorphine.

\section{CASE REPORT \#2 - LH}

A 20 year old male with a history of heroin abuse presented to the clinic for the first time with complaints of headache located directly behind both eyes that was associated with photophobia, cold sweats, abdominal pain, generalized body aches, itchiness, decreased sleep, and runny nose.

Further history revealed that the patient had been using heroin every day for one year, but stated he wanted to get clean. According to the patient, he used to smoke heroin 4 to 5 times a day. The patient further said that he got into the habit because of spending time in bad company. Prior to presentation at the clinic, he said he had smoked heroin the day before. The patient also had a history of marijuana abuse. He also had a one year history of smoking approximately 5 cigarettes per day, but was taking no other medications at the time, and had no other significant medical history.

Upon examination, the patient had moderate tenderness bilaterally over the ocular orbits. His chest was clear to auscultation, there was no organomegaly, and he had normal cranial nerve findings. There were no other significant physical findings.
The patient was started on Suboxone 2-0.5 mg tablets, and his instructions, per the Suboxone protocol, were to take two tablets twice a day for the first day, and then from the second day on, to take two tablets four times a day. When the patient presented to the clinic on the third day of his treatment with Suboxone, he reported that his cold sweats had gone away, and so had his runny nose. He still had a headache, graded at 3 out of 10 on the pain scale, and light still bothered his eyes a little. He also had a dull abdominal pain, also graded as 3 out of 10, and slightly achy elbows and knees. His dosage of Suboxone was increased at that visit to 4 tablets of $2-.5 \mathrm{mg} 3$ times a day.

The next time he was seen, four days after his second visit to the clinic, and on the seventh day of treatment, his headache and abdominal pain were graded as a 2-3 out of 10, and his achy joints at a 3 out of 10. A Normal Saline infusion was initiated and he was given ketamine $5 \mathrm{mg}$ IV bolus (repeated once) and 60mg of ketorolac IV bolus. Afterward, the patient reported complete resolution of the abdominal pain, and that his headache was reduced to a 1 out of 10 . The pain in his elbows had gone away, and his knee pain was nearly gone, as well, at a 1-2 out of 10. A follow up after two months since his first visit to the clinic revealed that patient was completely withdrawal free thus accomplishing another successful shift to buprenorphine.

\section{DISCUSSION}

Methadone has been used for over fifty years as a pharmaceutical of choice for opiate replacement in addicted or opioid-tolerant persons for the purpose of reducing and eventually discontinuing opiate drugs, as it is a long-acting full synthetic opioid agonist, binding to the $\mu$-opioid receptor. As it does not provide the "high" of short-acting opiates, it helps relieve the cravings without reinforcing the feelings and behavior of addiction.

Ketamine is a dissociative anaesthetic, interrupting the thalamocortical loop, reticular activating system, and limbic system to produce sensory blockade and reduce arousal and wakefulness. It is classified pharmacologically as an NMDA receptor antagonist. In one study, IV ketamine significantly suppressed the expression of precipitated opiate withdrawal and prevented significant rise in cardiovascular, respiratory, and neuroendocrine response [3]. There has been another report of using ketamine as a sedative in the case of a 2 year old who was dependent on opiates, with the effect that it alleviated symptoms of her withdrawal [2]. However, it has not been used in a methodical attempt to ameliorate the unpleasant side effects of abruptly discontinuing opiate use and precipitating into withdrawal. Our observations suggest that intravenous ketamine is more effective than any other medications in our patients for treating the acute withdrawal symptoms while switching to buprenorphine.

Naloxone and naltrexone are competitive antagonists at the mu, kappa, and sigma receptors. Buprenorphine is classified as a partial agonist. It has a high affinity, but low efficacy at the mu receptor where it has a partial effect upon binding. It also, however, possesses kappa receptor antagonist activity making it useful not only as analgesic, but also in opioid abuse avoidance, detoxification, and maintenance therapies [4]. Naloxone is added to sublingual 
buprenorphine to prevent the intravenous abuse of buprenorphine. The same product (sublingual buprenorphine) when used alone (i.e. without naloxone) is marketed as Subutex. Although the success of Suboxone is clear, once it has been started, there is still a period of 12-72 hours during which the patient suffers withdrawal symptoms due to abruptly ceasing opiate administration. In addition, patients who have chronic pain will experience exacerbation of pain symptoms when their regular opiates are quickly discontinued.

\section{CONCLUSION}

Our case reports demonstrates that IV ketamine can be a very effective treatment for the opioid withdrawal symptoms as well as for the pain that these patients experience while being transitioned to buprenorphine. However more research is needed in this area.

\section{ACKNOWLEDGEMENT}

None Declared

\section{CONFLICT OF INTEREST}

None Declared

\section{REFERENCES:}

[1] Stinus L, Le Moal M, Koob GF. Nucleus accumbens and amygdala are possible substrates for the aversive stimulus effects of opiate withdrawal. Neuroscience 1990; 37: 767-73.

[2] Ito $\mathrm{H}$, Sobue K, Hirate $\mathrm{H}$, et al. Use of ketamine to facilitate opioid withdrawal in a child. Anesthesiology 2006; 104: 1113.

[3] Jovaisa T, Laurinenas G, Vosylius S, Sipylaite J, Badaras R, Ivaskevicius J. Effects of ketamine on precipitated opiate withdrawal. Medicina (Kaunas) 2006; 42(8): 625-33.

[4] Helm S, Trescot AM, Colson J, Sehgal N, Silverman SM. Opioid antagonists, partial Agonists, and agonists/antagonists: the role of office-based detoxification. Pain Physician 2008; 11: 225-35. 